\title{
NON-AMENABLE TIGHT SQUEEZES BY KIRCHBERG ALGEBRAS
}

\author{
YUHEI SUZUKI
}

\begin{abstract}
We give a framework to produce $\mathrm{C}^{*}$-algebra inclusions with extreme properties. This gives the first constructive nuclear minimal ambient $\mathrm{C}^{*}$-algebras. We further obtain a purely infinite analogue of Dadarlat's modeling theorem on AF-algebras: Every Kirchberg algebra is rigidly and KK-equivalently sandwiched by non-nuclear $\mathrm{C}^{*}$-algebras without intermediate $\mathrm{C}^{*}$-algebras. Finally we reveal a novel property of Kirchberg algebras: They embed into arbitrarily wild $\mathrm{C}^{*}$-algebras as rigid maximal $\mathrm{C}^{*}$-subalgebras.
\end{abstract}

\section{INTRODUCTION}

Thanks to the recent progress in the classification theory of amenable $\mathrm{C}^{*}$-algebras, Elliott's classification program has been almost completed; see [58] for a recent survey. As crucial ideas and techniques have been developed in this theory (see e.g., [16], [30], [36], [41], [57], and references in [58]), a next natural attempt is applying the theory and its byproducts to understand the structure of simple $\mathrm{C}^{*}$-algebras beyond the classifiable class (e.g., the reduced non-amenable group $\mathrm{C}^{*}$-algebras).

To take the advantage of rich structures of classifiable $\mathrm{C}^{*}$-algebras to understand nonamenable $\mathrm{C}^{*}$-algebras, one possible natural strategy is to bridge two $\mathrm{C}^{*}$-algebras from each class via a tight inclusion. Indeed tight inclusions of operator algebras receive much attentions and are deeply studied by many hands because of their importance in the structure theory of operator algebras: see e.g., [21], [22], 29], [35], [38], [46], [47], 48]. Recent highlights are the breakthrough results on $\mathrm{C}^{*}$-simplicity [29], [7] (cf. [22]), in which tight inclusions are used to reduce problems on the reduced group $\mathrm{C}^{*}$-algebras to those of less complicated $\mathrm{C}^{*}$-algebras. This suggests the existence of boundary theory for general $\mathrm{C}^{*}$-algebras (cf. [38]). Other strategies based on expansions of $\mathrm{C}^{*}$-algebras also work successfully in the Baum-Connes conjecture [5], see e.g., 24], 25]. These significant results are motivations behind the present work.

The purpose of the present paper is to establish a new powerful framework to produce extreme examples of tight $\mathrm{C}^{*}$-algebra inclusions. We particularly consider the following three conditions coming from the three different viewpoints:

Algebraic side: absence of intermediate $\mathrm{C}^{*}$-algebras (maximality/minimality),

Topological side: KK-equivalence,

Order structural side: Hamana's operator system rigidity [20].

We note that the third condition is a crucial ingredient of [29], [7]. Several questions on the first condition were posed by Ge [17] for instance. The second condition is partly motivated by the Baum-Connes conjecture (cf. [24, [25]).

2010 Mathematics Subject Classification. Primary 46L55, Secondary 46L05, 46L07.

Key words and phrases. Kirchberg algebras, $\mathrm{C}^{*}$-dynamical systems, rigid inclusions. 
We now present the Main Theorem of this paper. Throughout the paper, denote by $\mathbb{F}_{\infty}$ a countable free group of infinite rank.

Main Theorem (see Proposition 4.4, Theorems 3.3, 4.8). Let $A$ be a simple unital separable purely infinite $C^{*}$-algebra. Let $\alpha: \mathbb{F}_{\infty} \curvearrowright A$ be an approximately inner $C^{*}$ dynamical system. Then there is an inner perturbation $\gamma$ of $\alpha$ with the following property: Any $C^{*}$-dynamical system $\beta: \mathbb{F}_{\infty} \curvearrowright B$ on a simple $C^{*}$-algebra $B$ with $B^{\beta} \neq 0$ gives a rigid inclusion $B \rtimes_{\mathrm{r}, \beta} \mathbb{F}_{\infty} \subset(A \otimes B) \rtimes_{\mathrm{r}, \gamma \otimes \beta} \mathbb{F}_{\infty}$ without intermediate $C^{*}$-algebras.

Note that the statement does not exclude the case that $B=\mathbb{C}$ and the case that $\alpha$ is the trivial action. Even in these specific cases, the theorem provides many $\mathrm{C}^{*}$-algebra inclusions of remarkable new features. The Main Theorem also sheds some light on $\mathrm{C}^{*}$ dynamical systems. Inner automorphisms are usually regarded as trivial objects in the study of automorphisms of (single) $\mathrm{C}^{*}$-algebras. In fact when two $\mathrm{C}^{*}$-dynamical systems are cocycle conjugate, their crossed product $\mathrm{C}^{*}$-algebras are isomorphic. However, the Main Theorem reveals that the associated $\mathrm{C}^{*}$-algebra inclusions can be changed drastically by inner automorphisms. It is also interesting to compare these phenomena with the remarkable rigidity phenomena on the crossed product algebra inclusions studied and conjectured by Neshveyev-Størmer [37] (see also the recent works [10], [54]).

Applications to Kirchberg algebras. Since it is fairly easy to construct free group $\mathrm{C}^{*}$-dynamical systems (because of the freeness), the Main Theorem has a wide range of applications. Furthermore, although it is not immediately apparent from the statement, the Main Theorem is successfully applied to arbitrary Kirchberg algebras. As a consequence, we obtain novel properties of Kirchberg algebras.

Recall that a $\mathrm{C}^{*}$-algebra is said to be a Kirchberg algebra if it is simple, separable, nuclear, and purely infinite. We refer the reader to the book [50] for basic facts and backgrounds on Kirchberg algebras. Some beautiful and rich features of Kirchberg algebras can be seen from the complete classification theorem of Kirchberg [30] and Phillips [41]. We also refer the reader to [27], 28] (cf. [13]) for a new interaction between algebraic topology and the symmetry structure of Kirchberg algebras.

As the first main consequence, we obtain the first constructive examples of nuclear minimal ambient $\mathrm{C}^{*}$-algebras. (Here constructive at least means that all constructions are elementary and concretely understandable and avoid the Baire category theorem.)

Theorem A. Let $\alpha: \mathbb{F}_{\infty} \curvearrowright A$ be a $C^{*}$-dynamical system on a simple separable nuclear $C^{*}$ algebra with $A^{\alpha} \neq 0$. Then the reduced crossed product $A \rtimes_{\mathrm{r}, \alpha} \mathbb{F}_{\infty}$ admits a KK-equivalent rigid embedding into a Kirchberg algebra without intermediate $C^{*}$-algebras.

Note that, in our previous work [52], we obtained partial results in specific cases (without KK-condition), which in particular gave the first examples of nuclear minimal ambient $\mathrm{C}^{*}$-algebras. However all constructions in [52] depend on the Baire category theorem (applied to the space of Cantor systems of $\mathbb{F}_{\infty}$ ). It is a novelty of the present paper that our new constructions are quite elementary and avoid the Baire category theorem.

The second main consequence is the following structure result on Kirchberg algebras. This theorem can be considered as a Kirchberg algebra analogue of Dadarlat's modeling theorem for AF-algebras [12]. Since Kirchberg algebras have no elementary inductive limit structure, our approach naturally differs from [12]. 
Theorem B. For every Kirchberg algebra $A$, there are $C^{*}$-algebra inclusions $B \subset A \subset C$ satisfying the following conditions.

- $B$ is non-nuclear, $C$ is non-exact, and both algebras are simple and purely infinite.

- The $C^{*}$-subalgebras $B \subset A$ and $A \subset C$ are rigid and maximal.

- These inclusions give KK-equivalences.

As the third application, we obtain the following ubiquitous property for some $\mathrm{C}^{*}$ algebras. Although the statement holds true for more general $\mathrm{C}^{*}$-algebras, we concentrate on the particularly interesting case.

Theorem C. Let $A$ be a unital Kirchberg algebra. Then for any unital separable $C^{*}$ algebra $B$, there exists an ambient $C^{*}$-algebra $C$ of $B$ with a faithful conditional expectation which also contains $A$ as a rigid maximal $C^{*}$-subalgebra.

It is easy to see from the proof that $\mathrm{C}_{\mathrm{r}}^{*}\left(\mathbb{F}_{\infty}\right)$ also has the same property. Moreover one can show that the free group factor $L\left(\mathbb{F}_{\infty}\right)$ has the analogous property by a similar method (Remark [5.3). It would be interesting to ask if the other free group factors $L\left(\mathbb{F}_{n}\right)$; $n=2,3, \ldots$ have the same property.

A key ingredient of the proofs is amenable actions of $\mathbb{F}_{\infty}$ on Kirchberg algebras [53], [54]. The existence of amenable actions (of non-amenable groups) on simple $\mathrm{C}^{*}$-algebras does not seem to have been believed for a long time (cf. [4, [9]) until [53]. (It is notable that such actions do not exist in the von Neumann algebra context; see [2], Corollary 4.3.) To exclude intermediate $\mathrm{C}^{*}$-algebras of the reduced crossed product inclusions, as stated in the Main Theorem, we perturb actions by inner automorphisms. In contrast to commutative $\mathrm{C}^{*}$-algebras, purely infinite simple $\mathrm{C}^{*}$-algebras have sufficiently many inner automorphisms (by Cuntz's result [11]; see Lemma 2.4 below). This provides amenable actions on Kirchberg algebras which sufficiently mix projections. (Note that by Zhang's theorem [60], purely infinite simple $\mathrm{C}^{*}$-algebras are of real rank zero. In particular they have plenty of nontrivial projections. This theorem plays a prominent role throughout the article.) Another important advantage of using Kirchberg algebras is their freedom of K-theory: in contrast to the fact that the K-groups of compact spaces are restricted (for instance their $\mathrm{K}^{0}$-groups must have a non-trivial order structure), there is no structural restriction on the K-groups of Kirchberg algebras. This leads to useful reduced crossed product decompositions of Kirchberg algebras by $\mathbb{F}_{\infty}$ (up to stable isomorphism) [54].

Organization of the paper. In Section 2, we develop techniques on inner perturbations of $\mathrm{C}^{*}$-dynamical systems. This provides $\mathrm{C}^{*}$-dynamical systems with extremely transitive properties. In Section 3, we study restrictions of intermediate objects of certain structures associated with $\mathrm{C}^{*}$-dynamical systems obtained in Section 2. In Section 4, we discuss rigidity properties of inclusions. In particular, after improving the constructions of inner perturbations in Section 2, we complete the proof of the Main Theorem. Finally, in Section 5, we prove the consequences of the Main Theorem (Theorems A to C).

To handle the non-unital case, we need technical results, which we discuss in the Appendix. As a byproduct, we extend the tensor splitting theorem [59], 61] (cf. [18]) to the non-unital case.

Finally, we remark that, except for some cases, the crossed product splitting theorem obtained in [54] is not available because of the failure of central freeness. Our method to 
exclude intermediate $\mathrm{C}^{*}$-algebras is a sophisticated version of the argument developed in our previous work [52]. Because of non-commutativity and K-theoretic obstructions, we need technical improvements.

For basic facts on $\mathrm{C}^{*}$-algebras and discrete groups, we refer the reader to the book [9]. For basic facts on K-theory and KK-theory, see the book [6].

Notations. Here we fix some notations. Notations not explained in the article should be very common in operator algebra theory.

- For $\epsilon>0$ and for two elements $x, y$ of a $\mathrm{C}^{*}$-algebra, denote by $x \approx_{\epsilon} y$ if $\|x-y\|<\epsilon$.

- The symbols ' $\otimes$ ', ' $\rtimes_{\mathrm{r}}$ ', ' $\rtimes_{\text {alg }}$ ' stand for the minimal tensor products (of $C^{*}$-algebras and completely bounded maps) and the reduced $\mathrm{C}^{*}$ - and algebraic crossed products respectively.

- For a $\mathrm{C}^{*}$-algebra $A$, denote by $A^{\mathrm{p}}, A_{+}$the set of projections and the cone of positive elements in $A$ respectively.

- For a unital $\mathrm{C}^{*}$-algebra $A$, denote by $A^{\mathrm{u}}$ the group of unitary elements in $A$.

- For a $\mathrm{C}^{*}$-algebra $A$, denote by $\mathcal{M}(A), Z(A), A^{* *}$ the multiplier algebra of $A$, the center of $A$, and the second dual of $A$ respectively.

- When there is an obvious $\mathrm{C}^{*}$-algebra embedding $A \rightarrow \mathcal{M}(B)$, we regard $A$ as a $\mathrm{C}^{*}$-subalgebra of $\mathcal{M}(B)$ via the obvious embedding. Such a situation often occurs in the tensor product, the free product, and the crossed product constructions.

- For the reduced crossed product $A \rtimes_{\mathrm{r}} \Gamma$ and $s \in \Gamma$, denote by $u_{s}$ its canonical implementing unitary element in $\mathcal{M}\left(A \rtimes_{\mathrm{r}} \Gamma\right)$.

- For the reduced crossed product $A \rtimes_{\mathrm{r}} \Gamma$, denote by $E: A \rtimes_{\mathrm{r}} \Gamma \rightarrow A$ the conditional expectation satisfying $E\left(a u_{s}\right)=0$ for all $a \in A$ and $s \in \Gamma \backslash\{e\}$ (called the canonical conditional expectation).

- For a $\mathrm{C}^{*}$-dynamical system $\alpha: \Gamma \curvearrowright A$, denote by $A^{\alpha}$ the fixed point algebra of $\alpha$ :

$$
A^{\alpha}:=\left\{a \in A: \alpha_{s}(a)=a \text { for all } s \in \Gamma\right\} .
$$

- For two $\mathrm{C}^{*}$-dynamical systems $\alpha: \Gamma \curvearrowright A$ and $\beta: \Gamma \curvearrowright B$, denote by $\alpha \otimes \beta$ the diagonal action of $\alpha$ and $\beta$, that is, the action $\Gamma \curvearrowright A \otimes B$ defined to be $(\alpha \otimes \beta)_{s}:=$ $\alpha_{s} \otimes \beta_{s}$ for $s \in \Gamma$.

- For a $\mathrm{C}^{*}$-algebra $A$, denote by 1 the unit of $\mathcal{M}(A)$.

- For a unital $\mathrm{C}^{*}$-algebra $A$, denote by $\mathbb{C}$ the subspace of $A$ spanned by 1 .

\section{INNER PERTURBATIONS OF $\mathrm{C}^{*}$-DYNAMICAL SYSTEMS}

In this section, we develop techniques on inner perturbations of free group $\mathrm{C}^{*}$-dynamical systems. This provides $\mathrm{C}^{*}$-dynamical systems with an extreme transitivity; see Proposition 2.5. The results in this section play crucial roles in the proof of the Main Theorem.

We first introduce the following metric spaces of projections in a $\mathrm{C}^{*}$-algebra.

Definition 2.1. Let $A$ be a $\mathrm{C}^{*}$-algebra. For $x_{1}, x_{2} \in \mathrm{K}_{0}(A)$, we define

$$
\mathrm{P}\left(A ; x_{1}, x_{2}\right):=\left\{\left(p_{1}, p_{2}\right) \in\left(A^{\mathrm{p}} \backslash\{0\}\right)^{2}:\left[p_{1}\right]_{0}=x_{1},\left[p_{2}\right]_{0}=x_{2}, p_{1} \perp p_{2}, p_{1}+p_{2} \neq 1\right\}
$$

(possibly empty). We equip $\mathrm{P}\left(A ; x_{1}, x_{2}\right)$ with the metric given by the $\mathrm{C}^{*}$-norm on $A \oplus A$. 
Note that each $\mathrm{P}\left(A ; x_{1}, x_{2}\right)$ is closed in $A \oplus A$. Observe that every automorphism $\alpha$ on $A$ which acts trivially on the $\mathrm{K}_{0}$-group induces an isometric homeomorphism

$$
\left(p_{1}, p_{2}\right) \mapsto\left(\alpha\left(p_{1}\right), \alpha\left(p_{2}\right)\right)
$$

on each $\mathrm{P}\left(A ; x_{1}, x_{2}\right)$.

In this paper we employ the following definition of amenability for $\mathrm{C}^{*}$-dynamical systems which is introduced by Anantharaman-Delaroche [3].

Definition 2.2 (see [3], Definition 4.1). A C $\mathrm{C}^{*}$-dynamical system $\alpha: \Gamma \curvearrowright A$ is said to be amenable if the induced action $\Gamma \curvearrowright Z\left(A^{* *}\right)$ is amenable in the von Neumann algebra sense.

Although there is another property of $\mathrm{C}^{*}$-dynamical systems called amenable (see e.g., [9]), in this paper amenability always means the property in Definition 2.2 without specified. In this paper, we do not use the definition directly, but use the following facts.

(1) It is clear from the definition that when one of $\mathrm{C}^{*}$-dynamical systems $\alpha, \beta$ of $\Gamma$ is amenable, so is $\alpha \otimes \beta$.

(2) When the underlying $\mathrm{C}^{*}$-algebra is nuclear, amenability of $\mathrm{C}^{*}$-dynamical systems is equivalent to the nuclearity of the reduced crossed product ([3], Theorem 4.5).

We give one more basic property of amenability. This immediately follows from the definition, but plays an important role in this paper. Before giving the statement, we recall and introduce a few definitions.

Recall that an automorphism $\alpha$ of a $\mathrm{C}^{*}$-algebra $A$ is said to be inner if there exists $u \in \mathcal{M}(A)^{\mathrm{u}}$ satisfying $\alpha(x)=\operatorname{ad}(u)(x):=u x u^{*}$ for all $x \in A$. Denote by $\operatorname{Inn}(A)$ the group of inner automorphisms of $A$. For two $\mathrm{C}^{*}$-dynamical systems $\alpha, \beta: \Gamma \curvearrowright A$, we say that $\beta$ is an inner perturbation of $\alpha$ if $\beta_{s} \circ \alpha_{s}^{-1} \in \operatorname{Inn}(A)$ for all $s \in \Gamma$. Note that, as $\operatorname{Inn}(A)$ forms a normal subgroup in the automorphism group of $A$, to check that $\beta$ is an inner perturbation of $\alpha$, we only need to confirm the condition on a generating set of $\Gamma$.

Lemma 2.3. Amenability of $C^{*}$-dynamical systems is stable under inner perturbations.

Proof. Inner automorphisms on a $\mathrm{C}^{*}$-algebra $A$ induce the identity map on $Z\left(A^{* *}\right)$.

We remark that $\operatorname{Inn}(A)$ in Lemma 2.3 is not replaceable by its pointwise norm closure (the group of approximately inner automorphisms). In fact, when the acting group is a non-commutative free group, by [33], any $\mathrm{C}^{*}$-dynamical system on a simple separable $\mathrm{C}^{*}$-algebra admits a non-amenable approximately inner perturbation (see the Proposition in [56] for details and an application).

We next recall the following basic observation. The proof is essentially contained in [11] and should be well-known but we include it for completeness.

Lemma 2.4. Let $A$ be a purely infinite simple $C^{*}$-algebra. Then for any $x_{1}, x_{2} \in \mathrm{K}_{0}(A)$, the induced action $\operatorname{Inn}(A) \curvearrowright \mathrm{P}\left(A ; x_{1}, x_{2}\right)$ is transitive.

Proof. Note first that in the unital case, the statement follows from [11, Section 1. To consider the non-unital case, we first show that each orbit of the action is open in $\mathrm{P}\left(A ; x_{1}, x_{2}\right)$. Let $\left(p_{1}, p_{2}\right),\left(q_{1}, q_{2}\right) \in \mathrm{P}\left(A ; x_{1}, x_{2}\right)$ be given. Assume that $\left\|p_{1}-q_{1}\right\|,\left\|p_{2}-q_{2}\right\|<1 / 12$. By Lemma 7.2.2 in [9], there is $u \in \mathcal{M}(A)^{\mathrm{p}}$ with $q_{1}=u p_{1} u^{*},\|1-u\|<1 / 3$. This implies 
$\left\|u p_{2} u^{*}-q_{2}\right\|<1$. Applying Lemma 7.2.2 in [9] to the projections $u p_{2} u^{*}, q_{2}$ in the $\mathrm{C}^{*}$ algebra $\left(1-q_{1}\right) \mathcal{M}(A)\left(1-q_{1}\right)$, we obtain $v \in \mathcal{M}(A)^{\mathrm{u}}$ with $v u p_{2} u^{*} v^{*}=q_{2}, v q_{1}=q_{1}$. Now it is clear that $\operatorname{ad}(v u)\left(p_{i}\right)=q_{i}$ for $i=1,2$.

By [60], $A$ admits a (not necessary increasing) approximate unit $\left(e_{j}\right)_{j \in J}$ consisting of projections. Thus for any $\left(p_{1}, p_{2}\right) \in \mathrm{P}\left(A ; x_{1}, x_{2}\right)$, by standard applications of functional calculus and the observation in the previous paragraph, for any sufficiently large $j \in J$, one can find $\alpha \in \operatorname{Inn}(A)$ with $\alpha\left(p_{1}+p_{2}\right) \lesseqgtr e_{j}$. This reduces the proof to the unital case, and thus completes the proof.

Now we are able to show the following result. We say that a group action $\Gamma \curvearrowright X$ on a topological space is minimal if all $\Gamma$-orbits are dense in $X$.

Proposition 2.5. Let $\alpha$ : $\mathbb{F}_{\infty} \curvearrowright A$ be a $C^{*}$-dynamical system on a separable purely infinite simple $C^{*}$-algebra $A$ whose induced action on $\mathrm{K}_{0}(A)$ is trivial. Then there exists an inner perturbation $\beta$ of $\alpha$ satisfying the following conditions.

(1) For any $x_{1}, x_{2} \in \mathrm{K}_{0}(A)$, the induced action $\mathbb{F}_{\infty} \curvearrowright \mathrm{P}\left(A ; x_{1}, x_{2}\right)$ of $\beta$ is minimal.

(2) Let $S_{\beta}$ denote the set of all $p \in A^{\mathrm{p}}$ whose stabilizer subgroup of $\beta$ contains at least two canonical generating elements of $\mathbb{F}_{\infty}$. Then $S_{\beta}$ is dense in $A^{\mathrm{p}}$ in norm.

Proof. Since any automorphism on $A$ which acts trivially on $\mathrm{K}_{0}(A)$ induces an isometric homeomorphism on each $\mathrm{P}\left(A ; x_{1}, x_{2}\right)$, to check condition (1), we only need to find a dense orbit in each $\mathrm{P}\left(A ; x_{1}, x_{2}\right)$.

For each $x_{1}, x_{2} \in \mathrm{K}_{0}(A)$, choose a dense sequence $\left(p\left[x_{1}, x_{2}, n, 1\right], p\left[x_{1}, x_{2}, n, 2\right]\right)_{n=1}^{\infty}$ in $\mathrm{P}\left(A ; x_{1}, x_{2}\right)$ such that each term appears at least twice in the sequence. Denote by $S$ the canonical generating set of $\mathbb{F}_{\infty}$. We fix a bijective map

$$
f: \mathrm{K}_{0}(A) \times \mathrm{K}_{0}(A) \times \mathbb{N} \times\{1,2\} \rightarrow S .
$$

For each $\left(x_{1}, x_{2}, n\right) \in \mathrm{K}_{0}(A) \times \mathrm{K}_{0}(A) \times \mathbb{N}$, choose $u\left[x_{1}, x_{2}, n, i\right] \in \mathcal{M}(A)^{\mathrm{u}} ; i=1,2$ satisfying

$$
\begin{aligned}
& \left(\operatorname{ad}\left(u\left[x_{1}, x_{2}, n, 1\right]\right) \circ \alpha_{f\left(x_{1}, x_{2}, n, 1\right)}\right)\left(p\left[x_{1}, x_{2}, 1, j\right]\right)=p\left[x_{1}, x_{2}, n, j\right], \\
& \left(\operatorname{ad}\left(u\left[x_{1}, x_{2}, n, 2\right]\right) \circ \alpha_{f\left(x_{1}, x_{2}, n, 2\right)}\right)\left(p\left[x_{1}, x_{2}, n, j\right]\right)=p\left[x_{1}, x_{2}, n, j\right],
\end{aligned}
$$

for $j=1,2$ (this is possible by Lemma 2.4). For $s \in S$, define

$$
\beta_{s}:=\operatorname{ad}\left(u\left[f^{-1}(s)\right]\right) \circ \alpha_{s} .
$$

This formula defines an inner perturbation $\beta$ of $\alpha$. It is clear from the choice of $u$ 's that $\beta$ satisfies the required conditions.

\section{TRAnsitivity CONDItIONS AND ABSENCE OF INTERMEDIATE OBJECTS}

In this section, we use conditions (1) and (2) in Proposition 2.5 to exclude certain intermediate objects. These two conditions can be seen as a non-commutative variant of the property $\mathcal{R}$ defined for Cantor systems in [52], Proposition 3.3. On the one hand, because the property $\mathcal{R}$ requires an extreme transitivity (seemingly opposite to amenability of topological dynamical systems), it seems hopeless to obtain a constructive amenable example. On the other hand, in contrast to this, we have already obtained constructive amenable $\mathrm{C}^{*}$-dynamical systems satisfying these two conditions, thanks to high non-commutativity of the underlying algebras. 
A ( $\mathbb{C}$-linear) subspace $X$ of a $\mathrm{C}^{*}$-algebra is said to be self-adjoint if $x^{*} \in X$ for all $x \in X$. For a $\mathrm{C}^{*}$-dynamical system $\alpha: \Gamma \curvearrowright A$, a subspace $X \subset A$ is said to be $\alpha$-invariant if it satisfies $\alpha_{s}(X)=X$ for all $s \in \Gamma$.

To study intermediate $\mathrm{C}^{*}$-algebras, we first show that, when the underlying algebra is simple and purely infinite, from condition (1), we obtain the best possible restriction on invariant closed subspaces. The reason why we need to study subspaces rather than just subalgebras is as follows. For a $C^{*}$-subalgebra $C$ of the reduced crossed product $A \rtimes_{\mathrm{r}, \alpha} \Gamma$ satisfying $u_{s} C u_{s}^{*}=C$ for all $s \in \Gamma$, the set $E(C)$ forms an $\alpha$-invariant self-adjoint subspace of $A$, but it is not necessary a subalgebra.

Proposition 3.1. Let $A$ be a purely infinite simple $C^{*}$-algebra. Let $\alpha: \mathbb{F}_{\infty} \curvearrowright A$ be a $C^{*}$ dynamical system which acts trivially on $\mathrm{K}_{0}(A)$ and satisfies condition (1) in Proposition 2.5. Then $0, \mathbb{C}, A$ are the only possible $\alpha$-invariant closed self-adjoint subspaces of $A$.

Proof. Let $X$ be an $\alpha$-invariant closed self-adjoint subspace of $A$ different from 0 and $\mathbb{C}$. It suffices to show that $X=A$. We first note that, in the unital case, the subspace $X+\mathbb{C}$ is also $\alpha$-invariant, closed, and self-adjoint. We observe that the equality $X+\mathbb{C}=A$ implies $X=A$. To see this, assume that $X+\mathbb{C}=A, X \neq A$, and denote by $\varphi$ the (nonzero bounded) linear functional on $A$ defined by the (Banach space) quotient map $A \rightarrow A / X \cong \mathbb{C}$. Then, since $X$ is $\alpha$-invariant, so is $\varphi$ (that is, $\varphi \circ \alpha_{s}=\varphi$ for all $\left.s \in \mathbb{F}_{\infty}\right)$. It follows from condition (1) that for any two projections $p_{1}, p_{2} \in A^{\mathrm{p}} \backslash\{0,1\}$ with $\left[p_{1}\right]_{0}=\left[p_{2}\right]_{0}$, we have $\varphi\left(p_{1}\right)=\varphi\left(p_{2}\right)$. Since $\varphi(1) \neq 0$, one can find $p \in A^{\mathrm{p}} \backslash\{0,1\}$ with $\varphi(p) \neq 0$. Choose pairwise orthogonal nonzero projections $\left(p_{n}\right)_{n=1}^{\infty}$ in $A$ satisfying $\left[p_{n}\right]_{0}=[p]_{0}$ for all $n \in \mathbb{N}$. Then for any $N \in \mathbb{N}$, we have $N|\varphi(p)|=\left|\varphi\left(\sum_{n=1}^{N} p_{n}\right)\right| \leq\|\varphi\|$. This is a contradiction. Thus, in the unital case, we only need to show the statement under the additional assumption that $\mathbb{C} \subsetneq X$.

We will prove $A^{\mathrm{p}} \subset X$ under this assumption, which implies $X=A$ by [60]. Choose a self-adjoint contractive element $h$ in $X \backslash \mathbb{C}$ whose spectrum contains 0 and 1 . Let $\epsilon>0$ and $p \in A^{\mathrm{p}} \backslash\{0,1\}$ be given. Since $A$ is of real rank zero [60] (see also [8]), there exist nonzero pairwise orthogonal projections $p_{1}, \ldots, p_{l}$ in $A$ and a sequence $\lambda_{1}, \ldots, \lambda_{l-1}, \lambda_{l}=1$ in $[-1,1] \subset \mathbb{R}$ satisfying

$$
h \approx_{\epsilon} \sum_{i=1}^{l} \lambda_{i} p_{i}, \quad \sum_{i=1}^{l} p_{i} \neq 1 .
$$

By splitting the last term $p_{l}$ into two new projections if necessary, we may assume that $\left[p_{l}\right]_{0}=[p]_{0}$. We put $G:=\left\{\alpha_{s}: s \in \mathbb{F}_{\infty}\right\}$ for short. Take $q, r_{1} \in A^{\mathrm{p}} \backslash\{0,1\}$ satisfying

$$
q \perp r_{1}, \quad q+r_{1} \perp \sum_{i=1}^{l} p_{i}, \quad[q]_{0}=\left[\sum_{i=1}^{l-1} p_{i}\right]_{0} .
$$

Next we fix a real number

$$
0<\delta<\min \left\{1, \frac{\epsilon-\left\|h-\sum_{i=1}^{l} \lambda_{i} p_{i}\right\|}{4 l}\right\} .
$$


By applying condition (1) to $\left(\sum_{i=1}^{l-1} p_{i}, p_{l}\right) \in \mathrm{P}\left(A ;[q]_{0},\left[p_{l}\right]_{0}\right)$, we obtain $\gamma_{1} \in G$ satisfying

$$
\sum_{i=1}^{l-1} \gamma_{1}\left(p_{i}\right) \approx_{\delta} q, \quad \gamma_{1}\left(p_{l}\right) \approx_{\delta} p_{l}
$$

By Lemma 7.2.2 (1) in [9], one can take $u \in \mathcal{M}(A)^{\mathrm{u}}$ satisfying

$$
\|u-1\|<4 \delta, \quad u\left(\sum_{i=1}^{l-1} \gamma_{1}\left(p_{i}\right)\right) u^{*}=q .
$$

Set $q_{i}:=u \gamma_{1}\left(p_{i}\right) u^{*}\left(\approx_{8 \delta} \gamma_{1}\left(p_{i}\right)\right)$ for $i=1, \ldots, l-1$. Then, since $\left|\lambda_{i}\right| \leq 1$ for all $i$, we have

$$
\sum_{i=1}^{l} \lambda_{i} \gamma_{1}\left(p_{i}\right) \approx_{8 l \delta} p_{l}+\sum_{i=1}^{l-1} \lambda_{i} q_{i}
$$

Set

$$
x_{2}:=\frac{1}{2} \sum_{i=1}^{l-1} \lambda_{i}\left(p_{i}+q_{i}\right) \in\left(1-p_{l}-r_{1}\right) A\left(1-p_{l}-r_{1}\right) .
$$

Note that $x_{2}$ is self-adjoint and $\left\|x_{2}\right\| \leq 1 / 2$. Since $4 l \delta+\left\|h-\sum_{i=1}^{l} \lambda_{i} p_{i}\right\|<\epsilon$, we obtain

$$
h_{2}:=\frac{1}{2}\left(h+\gamma_{1}(h)\right) \approx_{\epsilon} p_{l}+x_{2} .
$$

Next we apply the same argument to $p_{l}+x_{2}$ and $\epsilon-\left\|h_{2}-\left(p_{l}+x_{2}\right)\right\|$ instead of $h$ and $\epsilon$ (with the same $p_{l}$ ). As a result we obtain $\gamma_{2} \in G, r_{2} \in A^{\mathrm{p}} \backslash\{0,1\}$ with $r_{2} \perp p_{l}$, and a self-adjoint element $x_{3}$ in $\left(1-p_{l}-r_{2}\right) A\left(1-p_{l}-r_{2}\right)$ satisfying

$$
h_{3}:=\frac{1}{2}\left[h_{2}+\gamma_{2}\left(h_{2}\right)\right] \approx_{\epsilon} p_{l}+x_{3}, \quad\left\|x_{3}\right\| \leq \frac{1}{2^{2}} .
$$

Fix $N \in \mathbb{N}$ satisfying $2^{-(N-1)}<\epsilon$. By iterating this argument $N$ times, we finally obtain $h_{N} \in X$ and $x_{N} \in\left(1-p_{l}\right) A\left(1-p_{l}\right)$ satisfying

$$
h_{N} \approx_{\epsilon} p_{l}+x_{N}, \quad\left\|x_{N}\right\| \leq \frac{1}{2^{N-1}}<\epsilon .
$$

Choose $\gamma \in G$ satisfying $\gamma\left(p_{l}\right) \approx_{\epsilon} p$ (which exists by condition (1)). We then obtain $p \approx_{3 \epsilon} \gamma\left(h_{N}\right) \in X$. Since $\epsilon>0$ is arbitrary, we conclude $p \in X$.

Remark 3.2. In the stably finite case, one cannot expect to find actions satisfying the conclusion of Proposition 3.1. Indeed, let $A$ be a non-commutative $\mathrm{C}^{*}$-algebra. Then, for any non-empty set $S$ of tracial states on $A$, the subspace $\bigcap_{\alpha \in \operatorname{Aut}(A)} \bigcap_{\tau \in S} \operatorname{ker}(\tau \circ \alpha) \subset A$ is proper, closed, self-adjoint, and invariant under $\operatorname{Aut}(A)$. (Note that by the Hahn-Banach theorem, this subspace is nonzero.)

We now combine the subspace restriction obtained in Proposition 3.1 with condition (2) to effectively apply the Powers averaging argument [49], 23]. As a result, we obtain strong restrictions of some reduced crossed product inclusions associated with $\mathrm{C}^{*}$-dynamical systems obtained in Proposition 2.5. 
Theorem 3.3. Let $\alpha: \mathbb{F}_{\infty} \curvearrowright A$ be an action on a purely infinite simple $C^{*}$-algebra satisfying conditions (1) and (2) in Proposition 2.5. Let $\beta: \mathbb{F}_{\infty} \curvearrowright B$ be an action on a simple $C^{*}$-algebra with $B^{\beta} \neq 0$. Then $0, B \rtimes_{\mathrm{r}, \beta} \mathbb{F}_{\infty},(A \otimes B) \rtimes_{\mathrm{r}, \alpha \otimes \beta} \mathbb{F}_{\infty}$ are the only possible $C^{*}$-subalgebras of $(A \otimes B) \rtimes_{\mathrm{r}, \alpha \otimes \beta} \mathbb{F}_{\infty}$ invariant under multiplications by $B \rtimes_{\mathrm{r}, \beta} \mathbb{F}_{\infty}$.

Thus, when we additionally assume that $A$ is unital, the reduced crossed product inclusion $B \rtimes_{\mathrm{r}, \beta} \mathbb{F}_{\infty} \subset(A \otimes B) \rtimes_{\mathrm{r}, \alpha \otimes \beta} \mathbb{F}_{\infty}$ has no intermediate $\mathrm{C}^{*}$-algebras.

Proof. Let $C$ be a $C^{*}$-subalgebra of $(A \otimes B) \rtimes_{\mathrm{r}, \alpha \otimes \beta} \mathbb{F}_{\infty}$ as in the statement. We first consider the case that $E(C) \subset B$. When $A$ is non-unital, this implies $C=0$. When $A$ is unital, since $C=C u_{s}$ for all $s \in \mathbb{F}_{\infty}$, by Proposition 3.4 of [51], we have $C \subset B \rtimes_{\mathrm{r}, \beta} \mathbb{F}_{\infty}$. Since $B \rtimes_{\mathrm{r}, \beta} \mathbb{F}_{\infty}$ is simple $\left([23]\right.$, Theorem I), this yields $C=0$ or $C=B \rtimes_{\mathrm{r}, \beta} \mathbb{F}_{\infty}$.

We next consider the case $E(C) \not \subset B$. Observe that $E(C)$ is an $(\alpha \otimes \beta)$-invariant selfadjoint subspace of $A \otimes B$. Hence the elements of the form $\left(\operatorname{id}_{A} \otimes \varphi\right)(E(c))$, where $\varphi$ is a pure state on $B$ and $c \in C$, span an $\alpha$-invariant self-adjoint subspace of $A$. By (the easy part of) Theorem A.3, this subspace is not contained in $\mathbb{C}$. Therefore, by Proposition 3.1, for any $\epsilon>0$ and any $p \in A^{\mathrm{p}}$, one can choose pure states $\varphi_{1}, \ldots, \varphi_{n}$ on $B$ and $c_{1}, \ldots, c_{n} \in C \backslash\{0\}$ satisfying

$$
\sum_{i=1}^{n}\left(\operatorname{id}_{A} \otimes \varphi_{i}\right)\left(E\left(c_{i}\right)\right) \approx_{\epsilon} p
$$

By the Akemann-Anderson-Pedersen excision theorem [1] ([9], Theorem 1.4.10) (applied to each $\varphi_{i}$ ), for each $i=1, \ldots, n$, one can choose $b_{i} \in B_{+}$satisfying

$$
\left\|b_{i}\right\|=1, \quad b_{i} E\left(c_{i}\right) b_{i} \approx_{\epsilon / 2 n}\left[\left(\mathrm{id}_{A} \otimes \varphi_{i}\right)\left(E\left(c_{i}\right)\right)\right] \otimes b_{i}^{2} .
$$

We fix an element $b \in\left(B^{\beta}\right)_{+}$with $\|b\|=1$. By applying Lemma A.2 to each $b_{i}^{2}$ in $B$, we obtain finite sequences $\left(v_{i, j}\right)_{j=1}^{l(i)}, i=1, \ldots, n$, in $B$ satisfying

$$
\left\|\sum_{j=1}^{l(i)} v_{i, j} b_{i}^{2} v_{i, j}^{*}-b\right\|<\frac{\epsilon}{2 n\left\|c_{i}\right\|}, \quad \sum_{j=1}^{l(i)} v_{i, j} v_{i, j}^{*} \leq 1 .
$$

Set $x_{i, j}:=v_{i, j} b_{i} \in B \subset \mathcal{M}\left((A \otimes B) \rtimes_{\mathrm{r}, \alpha \otimes \beta} \mathbb{F}_{\infty}\right)$ for $i=1, \ldots, n$ and $j=1, \ldots, l(i)$. We then obtain

$$
E\left(\sum_{i=1}^{n} \sum_{j=1}^{l(i)} x_{i, j} c_{i} x_{i, j}^{*}\right)=\sum_{i=1}^{n} \sum_{j=1}^{l(i)} x_{i, j} E\left(c_{i}\right) x_{i, j}^{*} \approx_{\epsilon}\left[\sum_{i=1}^{n}\left(\mathrm{id}_{A} \otimes \varphi_{i}\right)\left(E\left(c_{i}\right)\right)\right] \otimes b \approx_{\epsilon} p \otimes b .
$$

Summarizing the result, we have shown that, for any $p \in A^{\mathrm{p}}$, any $b \in\left(B^{\beta}\right)_{+}$, and any $\epsilon>0$, there exists $c \in C$ satisfying

$$
E(c) \approx_{\epsilon} p \otimes b
$$

Fix $p \in A^{\mathrm{p}}, \epsilon>0, b \in\left(B^{\beta}\right)_{+} \backslash\{0\}$, and take $c \in C$ satisfying $E(c) \approx_{\epsilon} p \otimes b$. We further assume that the stabilizer subgroup of $p$ contains at least two canonical generating elements $s_{1}, s_{2}$ of $\mathbb{F}_{\infty}$. Choose $c_{0} \in(A \otimes B) \rtimes_{\text {alg }} \mathbb{F}_{\infty}$ satisfying $c_{0} \approx_{\epsilon} c, E\left(c_{0}\right)=p \otimes b$. We apply the Powers argument [49], [23] to $c_{0}-p \otimes b$ by using $s_{1}, s_{2}$ (cf. [52], Lemma 3.8). 
As a result, we obtain a sequence $g_{1}, \ldots, g_{m}$ in $\left\langle s_{1}, s_{2}\right\rangle$ (the subgroup generated by $s_{1}$ and $\left.s_{2}\right)$ satisfying

$$
\frac{1}{m} \sum_{i=1}^{m} u_{g_{i}} c_{0} u_{g_{i}}^{*} \approx_{\epsilon} p \otimes b .
$$

This implies

$$
p \otimes b \approx_{2 \epsilon} \frac{1}{m} \sum_{i=1}^{m} u_{g_{i}} c u_{g_{i}}^{*} \in C .
$$

Since $\epsilon>0$ is arbitrary, we conclude $p \otimes b \in C$. Since $\alpha$ satisfies condition (2), $A$ is of real rank zero [60], and $B$ is simple, we obtain $C=(A \otimes B) \rtimes_{\mathrm{r}, \alpha \otimes \beta} \mathbb{F}_{\infty}$.

\section{Rigidity PROPERTIES OF INCLUSIONS}

We establish a rigidity of automorphisms for inclusions obtained in Theorem 3.3. It is notable that the same property plays an important role in the proof of the Galois correspondence theorem of Izumi-Longo-Popa [26] (see also [35]). We then slightly modify Proposition 2.5 to give rigid inclusions. This completes the proof of the Main Theorem.

By using the spectral $M$-subspaces (see [40], Definition 8.1.3), we obtain the following result from Proposition 3.1.

Lemma 4.1. Let $\alpha: \mathbb{F}_{\infty} \curvearrowright A$ be a $C^{*}$-dynamical system as in Proposition 3.1. Assume that $A$ is unital. Then there is no non-trivial automorphism of $A$ commuting with $\alpha$.

Proof. Let $\beta$ be an automorphism of $A$ commuting with $\alpha$. Then for any closed subset $K \subset \widehat{\mathbb{Z}}$ with $K^{-1}=K$, the $M$-subspace $M^{\beta}(K)$ forms a closed self-adjoint $\alpha$-invariant subspace of $A$. Since $1 \in M^{\beta}(\{1\})$, by Theorem 8.1.4 (iv), (ix) of [40], we have $1 \notin M^{\beta}(K)$ whenever $1 \notin K$. By Proposition 3.1, this forces that $M^{\beta}(K)=0$ for any compact subset $K \subset \widehat{\mathbb{Z}} \backslash\{1\}$. Consequently, by Theorem 8.1.4 (iii), (vii), (viii), (ix) of [40], we have $M^{\beta}(\{1\})=A$. Corollary 8.1.8 in [40] now yields $\beta=\mathrm{id}_{A}$.

For a completely positive map $\Phi: A \rightarrow B$ between $\mathrm{C}^{*}$-algebras, when it extends to a completely positive map $\mathcal{M}(A) \rightarrow \mathcal{M}(B)$ which is strictly continuous on the unit ball, we denote by $\Phi^{\mathcal{M}}$ such a (unique) extension. Such an extension exists if $\Phi$ maps an approximate unit of $A$ to that of $B$; see Corollary 5.7 in 34]. It is obvious that all completely positive maps appearing below satisfy this condition.

Proposition 4.2. Let $\alpha, \beta$ be as in Theorem 3.3. Assume that $A$ is unital. Then the inclusion $C:=B \rtimes_{\mathrm{r}, \beta} \mathbb{F}_{\infty} \subset(A \otimes B) \rtimes_{\mathrm{r}, \alpha \otimes \beta} \mathbb{F}_{\infty}:=D$ has the following property. If two automorphisms $\gamma_{1}, \gamma_{2}$ of $D$ coincide on $C$, then $\gamma_{1}=\gamma_{2}$.

Proof. To prove the statement, it suffices to show the following claim. Any automorphism $\gamma$ on $D$ with $\left.\gamma\right|_{C}=\operatorname{id}_{C}$ must be trivial. Given such $\gamma$. We will show that $\gamma^{\mathcal{M}}(A)=A$. To see this, we first show that $E^{\mathcal{M}}\left(\gamma^{\mathcal{M}}(A)\right) \subset A$. Take $x \in E^{\mathcal{M}}\left(\gamma^{\mathcal{M}}(A)\right) \subset \mathcal{M}(A \otimes B)$. Then note that $x$ commutes with $B$ (by standard arguments on multiplicative domains). Therefore, for any state $\varphi$ on $A$, we have

$$
\left(\varphi \otimes \operatorname{id}_{B}\right)^{\mathcal{M}}(x) \in Z(\mathcal{M}(B))=\mathbb{C} .
$$


Here and below, we regard $\mathcal{M}(A)=A$ and $\mathcal{M}(B)$ as $\mathrm{C}^{*}$-subalgebras of $\mathcal{M}(A \otimes B)$ in the obvious way. This shows that, for any state $\psi$ on $B$,

$$
\left(\varphi \otimes \operatorname{id}_{B}\right)^{\mathcal{M}}\left(\left(\operatorname{id}_{A} \otimes \psi\right)^{\mathcal{M}}(x)\right)=\left(\operatorname{id}_{A} \otimes \psi\right)^{\mathcal{M}}\left(\left(\varphi \otimes \operatorname{id}_{B}\right)^{\mathcal{M}}(x)\right)=\left(\varphi \otimes \operatorname{id}_{B}\right)^{\mathcal{M}}(x) .
$$

Observe that the maps $\left(\varphi \otimes \mathrm{id}_{B}\right)^{\mathcal{M}} ; \varphi$ a state on $A$, separate the points of $\mathcal{M}(A \otimes B)$. Therefore we conclude

$$
x=\left(\operatorname{id}_{A} \otimes \psi\right)^{\mathcal{M}}(x) \in A .
$$

Since $\gamma^{\mathcal{M}}\left(u_{s}\right)=u_{s}$ for all $s \in \mathbb{F}_{\infty}$, the unital completely positive map

$$
\left.\left(E^{\mathcal{M}} \circ \gamma^{\mathcal{M}}\right)\right|_{A}: A \rightarrow A
$$

is $\mathbb{F}_{\infty}$-equivariant. Proposition 3.1 shows that $E^{\mathcal{M}}\left(\gamma^{\mathcal{M}}(A)\right)$ is dense in $A$.

Fix $b \in\left(B^{\beta}\right)_{+} \backslash\{0\}$ with $\|b\|=1$ and $\epsilon>0$. Let $p \in A^{\text {p }}$ be a projection whose stabilizer subgroup of $\alpha$ contains at least two canonical generating elements $s_{1}, s_{2}$ of $\mathbb{F}_{\infty}$. Choose $x \in A$ satisfying $E^{\mathcal{M}}\left(\gamma^{\mathcal{M}}(x)\right) \approx_{\epsilon} p$. By applying the Powers argument [49], 23] to $\gamma^{\mathcal{M}}(x) b=\gamma(x \otimes b) \in D$ by using $s_{1}$ and $s_{2}$ (cf. the proof of Theorem 3.3 or [52, Lemma $3.8)$, we obtain a sequence $g_{1}, \ldots, g_{n}$ in $\left\langle s_{1}, s_{2}\right\rangle$ satisfying

$$
p \otimes b \approx_{\epsilon} \frac{1}{n} \sum_{i=1}^{n} u_{g_{i}} \gamma(x \otimes b) u_{g_{i}}^{*}=\frac{1}{n} \sum_{i=1}^{n} \gamma\left(\alpha_{g_{i}}(x) \otimes b\right) .
$$

Since $\epsilon>0$ is arbitrary, we obtain $p \otimes b \in \gamma(A \otimes b)$. (Note that $\gamma$ is isometric hence $\gamma(A \otimes b)$ is closed in $D$.) By condition (2) of $\alpha$ and [60], we obtain

$$
A \otimes b \subset \gamma(A \otimes b)=\gamma^{\mathcal{M}}(A) b .
$$

By Lemma A.2, one can choose a net $\left(\left(v_{i, \lambda}\right)_{i=1}^{n(\lambda)}\right)_{\lambda \in \Lambda}$ of finite sequences in $B$ satisfying

$$
\begin{gathered}
\sum_{i=1}^{n(\lambda)} v_{i, \lambda} v_{i, \lambda}^{*} \leq 1 \quad \text { for all } \lambda \in \Lambda, \\
\lim _{\lambda \in \Lambda} \sum_{i=1}^{n(\lambda)} v_{i, \lambda} b v_{i, \lambda}^{*}=1 \quad \text { in the strict topology of } \mathcal{M}(D) .
\end{gathered}
$$

Now for any $a \in A$, choose $x \in A$ with $a \otimes b=\gamma^{\mathcal{M}}(x) b$. Then, for any $\lambda \in \Lambda$,

$$
a \otimes\left(\sum_{i=1}^{n(\lambda)} v_{i, \lambda} b v_{i, \lambda}^{*}\right)=\gamma^{\mathcal{M}}(x)\left(\sum_{i=1}^{n(\lambda)} v_{i, \lambda} b v_{i, \lambda}^{*}\right) .
$$

By letting $\lambda$ tend to infinity, we obtain $a=\gamma^{\mathcal{M}}(x)$. Applying the same argument to $\gamma^{-1}$, we obtain $A=\gamma^{\mathcal{M}}(A)$. Thus $\left.\gamma^{\mathcal{M}}\right|_{A}$ defines an automorphism on $A$. Since $\gamma^{\mathcal{M}}\left(u_{s}\right)=u_{s}$ for all $s \in \mathbb{F}_{\infty}$, the automorphism $\left.\gamma^{\mathcal{M}}\right|_{A}$ commutes with $\alpha$. Lemma 4.1 therefore implies $\left.\gamma^{\mathcal{M}}\right|_{A}=\operatorname{id}_{A}$. Since $A \cdot C$ generates $D$, we conclude $\gamma=\operatorname{id}_{D}$.

We now construct rigid inclusions. We first recall the definition.

Definition 4.3 ([20], Definition 2.4). An inclusion $A \subset B$ of $\mathrm{C}^{*}$-algebras is said to be rigid if the identity map $\operatorname{id}_{B}$ is the only completely positive map $\Phi: B \rightarrow B$ satisfying $\left.\Phi\right|_{A}=\mathrm{id}_{A}$. 
By slightly modifying Proposition 2.5 (under a stronger assumption), we obtain $\mathrm{C}^{*}$ dynamical systems satisfying a stronger condition which is useful to study the rigidity of associated inclusions.

For a separable $\mathrm{C}^{*}$-algebra $A$, when we equip the automorphism group $\operatorname{Aut}(A)$ of $A$ with the point-norm topology, it forms a Polish group. (The point-norm topology of $\operatorname{Aut}(A)$ is the weakest topology on $\operatorname{Aut}(A)$ making the evaluation maps $\alpha \mapsto \alpha(a) \in A$ norm continuous for all $a \in A$.) Indeed, take a dense sequence $\left(a_{n}\right)_{n=1}^{\infty}$ in the unit ball of $A$. Then it is not hard to see that the metric $d$ on $\operatorname{Aut}(A)$ given by

$$
d(\alpha, \beta):=\sum_{n=1}^{\infty} \frac{1}{2^{n}}\left(\left\|\alpha\left(a_{n}\right)-\beta\left(a_{n}\right)\right\|+\left\|\alpha^{-1}\left(a_{n}\right)-\beta^{-1}\left(a_{n}\right)\right\|\right) ; \quad \alpha, \beta \in \operatorname{Aut}(A),
$$

confirms the statement. Denote by $\overline{\operatorname{Inn}}(A)$ the closure of the inner automorphism group $\operatorname{Inn}(A)$ in $\operatorname{Aut}(A)$. We say that a $\mathrm{C}^{*}$-dynamical system $\alpha: \Gamma \curvearrowright A$ is pointwise approximately inner if $\alpha_{s} \in \overline{\operatorname{Inn}}(A)$ for all $s \in \Gamma$.

Proposition 4.4. Let $\alpha: \mathbb{F}_{\infty} \curvearrowright A$ be a pointwise approximately inner $C^{*}$-dynamical system on a separable purely infinite simple $C^{*}$-algebra $A$. Then there exists an inner perturbation $\beta$ of $\alpha$ satisfying the following conditions.

(1) The set $\left\{\beta_{s}: s \in \mathbb{F}_{\infty}\right\}$ is dense in $\overline{\operatorname{Inn}}(A)$.

(2) Let $S_{\beta}$ denote the set of all $p \in A^{\mathrm{p}}$ whose stabilizer subgroup of $\beta$ contains at least two canonical generating elements of $\mathbb{F}_{\infty}$. Then $S_{\beta}$ is dense in $A^{\mathrm{p}}$ in norm.

Proof. We split the canonical generating set $S$ of $\mathbb{F}_{\infty}$ into two infinite subsets: $S=S_{1} \sqcup S_{2}$. We first perturb $\alpha_{s} ; s \in S_{2}$ by inner automorphisms as in the proof of Proposition 2.5 to ensure condition (2). We next choose a dense sequence $\left(\gamma_{n}\right)_{n=1}^{\infty}$ in $\overline{\operatorname{Inn}}(A)$. Fix a bijective map $f: \mathbb{N} \times \mathbb{N} \rightarrow S_{1}$. Since each $\alpha_{s}$ is approximately inner, there exist $v_{s} \in \mathcal{M}(A)^{\mathrm{u}}$; $s \in S_{1}$, satisfying

$$
\lim _{m \rightarrow \infty} \operatorname{ad}\left(v_{f(n, m)}\right) \circ \alpha_{f(n, m)}=\gamma_{n} \quad \text { for all } n \in \mathbb{N} .
$$

These unitary elements define the desired inner perturbation of $\alpha$.

Corollary 4.5. There is an amenable action of $\mathbb{F}_{\infty}$ on the Cuntz algebra $\mathcal{O}_{\infty}$ satisfying conditions (1) and (2) in Proposition 4.4.

Proof. Recall from the proof of Theorem 5.1 of [54] that $\mathbb{F}_{\infty}$ admits an amenable action $\alpha$ on $\mathcal{O}_{\infty}$. It follows from the construction that $\alpha$ is pointwise approximately inner. Now applying Proposition 4.4 (and Lemma 2.3) to $\alpha$, we obtain the desired action.

Remark 4.6. It follows from Lemma 2.4 that condition (1) of Proposition 4.4 is stronger than condition (1) of Proposition 2.5.

Lemma 4.7. Let $\alpha: \mathbb{F}_{\infty} \curvearrowright A$ be an action on a unital purely infinite simple $C^{*}$-algebra satisfying condition (1) in Proposition 4.4. Then there is no $\mathbb{F}_{\infty}$-equivariant unital completely positive map $\Phi: A \rightarrow A$ other than $\operatorname{id}_{A}$ (that is, $\mathbb{C} \subset A$ is $\mathbb{F}_{\infty}$-rigid).

Proof. Let $\Phi$ be as in the statement. By the assumption on $\alpha$, all inner automorphisms are in the closure of $\left\{\alpha_{s}: s \in \mathbb{F}_{\infty}\right\}$ in $\operatorname{Aut}(A)$. Therefore, for any $u \in A^{\mathrm{u}}$ and any $x \in A$, we have $\Phi\left(u x u^{*}\right)=u \Phi(x) u^{*}$. Applying the equality to $x=p \in A^{\mathrm{p}} \backslash\{0,1\}$ and unitary 
elements $u$ in $p A p \oplus(1-p) A(1-p) \subset A$, we obtain $\Phi(p)=u \Phi(p) u^{*}$. Thus $\Phi(p)$ commutes with $p A p \oplus(1-p) A(1-p)$. Note that since $A$ is simple, so are $p A p$ and $(1-p) A(1-p)$. We therefore obtain

$$
\Phi(p)=\lambda_{1} p+\lambda_{2}(1-p) \quad \text { for some } \lambda_{1}, \lambda_{2} \geq 0 .
$$

We will show that $\lambda_{2}=0$. Take $v \in A^{\mathrm{u}}$ which satisfies $q:=v p v^{*} \lesseqgtr 1-p$ (see [11]). Then

$$
\Phi(q)=v \Phi(p) v^{*}=\lambda_{1} q+\lambda_{2}(1-q) .
$$

This yields

$$
\Phi(p+q)=\lambda_{1}(p+q)+\lambda_{2}(1-p-q)+\lambda_{2}=\left(\lambda_{1}+\lambda_{2}\right)(p+q)+2 \lambda_{2}(1-p-q) .
$$

By iterating this argument, for any $N \in \mathbb{N}$, one can find $r_{N} \in A^{\mathrm{p}} \backslash\{0,1\}$ satisfying

$$
\Phi\left(r_{N}\right)=\left[\lambda_{1}+\left(2^{N}-1\right) \lambda_{2}\right] r_{N}+2^{N} \lambda_{2}\left(1-r_{N}\right) .
$$

Since $\Phi$ is contractive, this forces $\lambda_{2}=0$. Thus $\Phi(p) \leq p$ for all $p \in A^{\mathrm{p}}$. Since $\Phi$ is unital, these inequalities imply $\Phi(p)=p$ for all $p \in A^{\mathrm{p}}$. Since $A^{\mathrm{p}}$ spans a dense subspace of $A$ 60], we conclude $\Phi=\mathrm{id}_{A}$.

Theorem 4.8. Let $\alpha: \mathbb{F}_{\infty} \curvearrowright A$ be a $C^{*}$-dynamical system on a unital purely infinite simple $C^{*}$-algebra satisfying condition (1) in Proposition 4.4. Let $\beta: \mathbb{F}_{\infty} \curvearrowright B$ be a $C^{*}$ dynamical system on a simple $C^{*}$-algebra. Then the inclusion

$$
C:=B \rtimes_{\mathrm{r}, \beta} \mathbb{F}_{\infty} \subset(A \otimes B) \rtimes_{\mathrm{r}, \alpha \otimes \beta} \mathbb{F}_{\infty}:=D
$$

is rigid.

Proof. Let $\Phi: D \rightarrow D$ be a completely positive map with $\left.\Phi\right|_{C}=\mathrm{id}_{C}$. Observe that $C$ contains an approximate unit of $D$. Hence by Corollary 5.7 of [34], the $\Phi$ has a strictly continuous extension $\Phi^{\mathcal{M}}: \mathcal{M}(D) \rightarrow \mathcal{M}(D)$. Since $\left.\Phi\right|_{B}=\mathrm{id}_{B}$, standard arguments on multiplicative domains show that

$$
\left(E^{\mathcal{M}} \circ \Phi^{\mathcal{M}}\right)(A) \subset \mathcal{M}(A \otimes B) \cap B^{\prime}=A .
$$

(For the proof of the last equality, see the proof of Proposition 4.2.) Since $\Phi^{\mathcal{M}}\left(u_{s}\right)=u_{s}$ for all $s \in \mathbb{F}_{\infty}$, the unital completely positive map

$$
\left.\left(E^{\mathcal{M}} \circ \Phi^{\mathcal{M}}\right)\right|_{A}: A \rightarrow A
$$

is $\mathbb{F}_{\infty}$-equivariant. Therefore Lemma 4.7 implies $\left.\left(E^{\mathcal{M}} \circ \Phi^{\mathcal{M}}\right)\right|_{A}=\mathrm{id}_{A}$. Observe that for any $u \in A^{\mathrm{u}}$ and any $x \in \mathcal{M}(D)$ satisfying $E^{\mathcal{M}}(x)=0$, we have

$$
\|u+x\|^{2} \geq\left\|E^{\mathcal{M}}\left((u+x)^{*}(u+x)\right)\right\|=1+\left\|E^{\mathcal{M}}\left(x^{*} x\right)\right\| .
$$

Since $E^{\mathcal{M}}: \mathcal{M}(D) \rightarrow \mathcal{M}(A \otimes B)$ is faithful, we obtain $\|u+x\|>\|u\|$ unless $x=0$. As both $E^{\mathcal{M}}$ and $\Phi^{\mathcal{M}}$ are contractive, the equality $\left.\left(E^{\mathcal{M}} \circ \Phi^{\mathcal{M}}\right)\right|_{A^{u}}=\operatorname{id}_{A^{u}}$ implies that $\left.\Phi^{\mathcal{M}}\right|_{A^{\mathrm{u}}}=\mathrm{id}_{A^{\mathrm{u}}}$. Since $A^{\mathrm{u}} \cdot C$ spans a dense subspace of $D$, we conclude $\Phi=\mathrm{id}_{D}$.

Now by combining Proposition 4.4 and Theorems 3.3, 4.8, we obtain the Main Theorem.

Before closing this section, we record the following elementary lemma on rigidity of $\mathrm{C}^{*}$-algebra inclusions. This lemma will be used in the next section.

Lemma 4.9. Let $A \subset B$ be a rigid inclusion of unital purely infinite simple $C^{*}$-algebras. Let $p \in A^{\mathrm{p}}$. Then the inclusion $p A p \subset p B p$ is also rigid. 
Proof. Assume that the inclusion $p A p \subset p B p$ is not rigid. Take a completely positive map $\Phi: p B p \rightarrow p B p$ satisfying $\left.\Phi\right|_{p A p}=\operatorname{id}_{p A p}$ and $\Phi \neq \operatorname{id}_{p B p}$. Choose $v \in A$ satisfying $v^{*} v=1$, $v v^{*} \leq p$. Define $\Psi: B \rightarrow B$ to be $\Psi(x):=v^{*} \Phi\left(v x v^{*}\right) v, x \in B$. Then for any $x \in p B p$, since $v p \in p A p$, we obtain

$$
\Psi(x)=v^{*} \Phi\left(v p x p v^{*}\right) v=v^{*} v p \Phi(x) p v^{*} v=\Phi(x) .
$$

In particular, $\Psi \neq \mathrm{id}_{B}$. Also, for any $a \in A$, as $v a v^{*} \in p A p$, we have

$$
\Psi(a)=v^{*} \Phi\left(v a v^{*}\right) v=v^{*} v a v^{*} v=a .
$$

In summary, we obtain $\left.\Psi\right|_{A}=\mathrm{id}_{A}, \Psi \neq \mathrm{id}_{B}$. Thus the inclusion $A \subset B$ is not rigid.

\section{Applichtions to Kirchberg algebras: proofs of Theorems a to C}

We now apply the Main Theorem to obtain the main results.

Proof of Theorem $A$. Let $\beta: \mathbb{F}_{\infty} \curvearrowright \mathcal{O}_{\infty}$ be an action obtained in Corollary 4.5. We show that $B:=A \rtimes_{\mathrm{r}, \alpha} \mathbb{F}_{\infty} \subset C:=\left(A \otimes \mathcal{O}_{\infty}\right) \rtimes_{\mathrm{r}, \alpha \otimes \beta} \mathbb{F}_{\infty}$ gives the desired ambient $\mathrm{C}^{*}$-algebra.

We first show that $C$ is a Kirchberg algebra. Clearly $C$ is separable. Since $A \otimes \mathcal{O}_{\infty}$ is simple, purely infinite, and $\alpha \otimes \beta$ is outer (because of its amenability and the fact that $\mathbb{F}_{\infty}$ has no non-trivial amenable normal subgroup), it follows from Kishimoto's theorem [32] that $C$ is purely infinite and simple (see e.g. Lemma 6.3 of [55] for details). Since $A \otimes \mathcal{O}_{\infty}$ is nuclear, so is $C$ by the amenability of $\alpha \otimes \beta$. Thus $C$ is a Kirchberg algebra.

By Theorem 3.3 , the inclusion indeed has no intermediate $\mathrm{C}^{*}$-algebras. By Theorem 4.8, the inclusion is rigid. Since the inclusion $\mathbb{C} \subset \mathcal{O}_{\infty}$ is a KK-equivalence [11], [44], so is $A \subset A \otimes \mathcal{O}_{\infty}$. Now it follows from Theorem 16 of [42] (see also [45]) that the inclusion $B \subset C$ is a KK-equivalence. (Proof: We apply the exact sequences in Theorem 16 of [42] to a fixed free action of $\mathbb{F}_{\infty}$ on a countable tree. Observe that for any countable set $I$, the inclusion $\bigoplus_{I} A \subset \bigoplus_{I}\left(A \otimes \mathcal{O}_{\infty}\right)$ is a KK-equivalence. By the Five Lemma and naturality of the exact sequences, the inclusion map $\iota: B \rightarrow C$ induces group isomorphisms

$$
\varphi: \mathrm{KK}(C, B) \rightarrow \mathrm{KK}(B, B), \quad \psi: \mathrm{KK}(C, B) \rightarrow \mathrm{KK}(C, C) .
$$

Put $x:=\varphi^{-1}\left(1_{B}\right), y:=\psi^{-1}\left(1_{C}\right)$. It then follows from the definition that

$$
[\iota] \hat{\otimes}_{C} x=\varphi(x)=1_{B}, \quad y \hat{\otimes}_{B}[\iota]=\psi(y)=1_{C} .
$$

Thus $x=y$ and $\iota$ is a KK-equivalence.)

Remark 5.1. Recall that any discrete exact group $\Gamma$ admits an amenable action on a unital purely infinite simple nuclear $\mathrm{C}^{*}$-algebra of density character $\sharp \Gamma$; see the proof of Proposition B in 53]. For the existence of a nuclear minimal ambient $\mathrm{C}^{*}$-algebra, our construction works for groups of the form $\mathbb{F}_{\Lambda} * \Lambda$ for any infinite group $\Lambda$ with the approximation property [25]. (However the resulting ambient algebras would be mysterious, cf. [55]). In particular, the reduced group $\mathrm{C}^{*}$-algebras of uncountable free groups admit a nuclear minimal ambient $\mathrm{C}^{*}$-algebra.

Proof of Theorem $B$. Let $A$ be a Kirchberg algebra. We have constructed, in the proof of the Proposition in [56] (see also the proof of Theorem 5.1 in [54]), an action $\alpha: \mathbb{F}_{\infty} \curvearrowright C$ on a unital Kirchberg algebra $C$ in the bootstrap class whose reduced crossed product $C \rtimes_{\mathrm{r}, \alpha} \mathbb{F}_{\infty}$ is non-nuclear, purely infinite simple, and KK-equivalent to $\mathcal{O}_{\infty}$. Let $\beta: \mathbb{F}_{\infty} \curvearrowright$ $\mathcal{O}_{\infty}$ be an amenable action obtained in Corollary 4.5. As shown in the proofs of the 
Proposition in [56] and Theorem 5.1 in [54] (by using [30], [41]), the crossed product $\left(C \otimes \mathcal{O}_{\infty}\right) \rtimes_{\mathrm{r}, \alpha \otimes \beta} \mathbb{F}_{\infty}$ is stably isomorphic to $\mathcal{O}_{\infty}$. Fix a projection

$$
p \in C \rtimes_{\mathrm{r}, \alpha} \mathbb{F}_{\infty}
$$

which generates $\mathrm{K}_{0}\left(\left(C \otimes \mathcal{O}_{\infty}\right) \rtimes_{\mathrm{r}, \alpha \otimes \beta} \mathbb{F}_{\infty}\right) \cong \mathbb{Z}$. (This is possible by [45] and [11].) Denote by 1 the trivial action of $\mathbb{F}_{\infty}$ on $A$. Then by the Kirchberg $\mathcal{O}_{\infty}$-absorption theorem [31], the corner $p\left[\left(A \otimes C \otimes \mathcal{O}_{\infty}\right) \rtimes_{\mathrm{r}, 1 \otimes \alpha \otimes \beta} \mathbb{F}_{\infty}\right] p$ is isomorphic to $A$. The desired subalgebra of $A \cong p\left[\left(A \otimes C \otimes \mathcal{O}_{\infty}\right) \rtimes_{\mathrm{r}, 1 \otimes \alpha \otimes \beta} \mathbb{F}_{\infty}\right] p$ is given by

$$
p\left[(A \otimes C) \rtimes_{\mathrm{r}, 1 \otimes \alpha} \mathbb{F}_{\infty}\right] p \subset p\left[\left(A \otimes C \otimes \mathcal{O}_{\infty}\right) \rtimes_{\mathrm{r}, 1 \otimes \alpha \otimes \beta} \mathbb{F}_{\infty}\right] p .
$$

Indeed, by Theorem 3.3 and [54], Lemma 5.2, the inclusion has no intermediate $\mathrm{C}^{*}$ algebras. By Theorem 4.8 and Lemma 4.9, the inclusion is rigid. Note that the corner $p\left[(A \otimes C) \rtimes_{\mathrm{r}, 1 \otimes \alpha} \mathbb{F}_{\infty}\right] p$ is isomorphic to $A \otimes p\left(C \rtimes_{\mathrm{r}, \alpha} \mathbb{F}_{\infty}\right) p$, which is not nuclear by the choice of $\alpha$. By [42] or [45] (see the proof of Theorem $\mathrm{A}$ for details), the inclusion gives a KK-equivalence.

We next construct an ambient non-exact $\mathrm{C}^{*}$-algebra of $p\left[\left(A \otimes C \otimes \mathcal{O}_{\infty}\right) \rtimes_{\mathrm{r}, 1 \otimes \alpha \otimes \beta} \mathbb{F}_{\infty}\right] p \cong$ $A$ as in the statement. We first take a non-exact unital simple separable $\mathrm{C}^{*}$-algebra $D_{0}$ such that the inclusion $\mathbb{C} \subset D_{0}$ is a KK-equivalence. (Example: Take a unital non-exact separable $\mathrm{C}^{*}$-algebra $P_{0}$. Set $P:=\left\{f \in C\left([0,1]^{2}, P_{0}\right): f(t, 0) \in \mathbb{C}\right.$ for all $\left.t \in[0,1]\right\}$. Note that $P$ is non-exact and homotopy equivalent to $\mathbb{C}$. Take a faithful state $\varphi$ on $P$ satisfying the conditions in Theorem 2 of [15]. By Exercise 4.8.1 in [9], there is a Hilbert $P$-bimodule whose Toeplitz-Pimsner algebra $D_{0}[42$ is isomorphic to the reduced free product $(P, \varphi) *(\mathcal{T}, \omega)$. Here $\mathcal{T}$ is the Toeplitz algebra and $\omega$ is a non-degenerate state on $\mathcal{T}$. By Theorem 4.4 of [43], the inclusion $P \subset D_{0}$ is a KK-equivalence. By Theorem 2 of [15], $D_{0}$ is simple. Thus $D_{0}$ gives the desired $\mathrm{C}^{*}$-algebra.) Set $D:=D_{0} \otimes \mathcal{O}_{\infty}$. Then $D$ is separable, purely infinite, simple, and the inclusion $\mathbb{C} \subset D$ gives a KK-equivalence. By applying Proposition 4.4 to the trivial action $\mathbb{F}_{\infty} \curvearrowright D$, we obtain an (inner) action $\gamma: \mathbb{F}_{\infty} \curvearrowright D$ satisfying conditions (1), (2) in Proposition 4.4. By the same reasons as in the previous paragraph, the inclusion

$$
p\left[\left(A \otimes C \otimes \mathcal{O}_{\infty}\right) \rtimes_{\mathrm{r}, 1 \otimes \alpha \otimes \beta} \mathbb{F}_{\infty}\right] p \subset p\left[\left(A \otimes C \otimes \mathcal{O}_{\infty} \otimes D\right) \rtimes_{\mathrm{r}, 1 \otimes \alpha \otimes \beta \otimes \gamma} \mathbb{F}_{\infty}\right] p
$$

is rigid, gives a KK-equivalence, and has no intermediate $\mathrm{C}^{*}$-algebras. The non-exactness of the largest $\mathrm{C}^{*}$-algebra is obvious. Finally, by Kirchberg's theorem ([50], Theorem 4.1.10 (i)), all these $\mathrm{C}^{*}$-algebras are purely infinite.

Remark 5.2. By a similar method to the Proposition in [56] (by using [39]), one can arrange the smallest algebra in Theorem B not having the completely bounded approximation property (see Section 12.3 of [9] for the definition).

Proof of Theorem C. Recall that in the proof of [54], Theorem 5.1, we obtained an amenable action $\alpha: \mathbb{F}_{\infty} \curvearrowright D$ on a unital Kirchberg algebra and a projection $p \in D$ such that $p\left(D \rtimes_{\mathrm{r}, \alpha} \mathbb{F}_{\infty}\right) p$ is isomorphic to $\mathcal{O}_{\infty}$. Denote by $1: \mathbb{F}_{\infty} \curvearrowright A$ the trivial action on $A$. By the Kirchberg $\mathcal{O}_{\infty}$-absorption theorem [31], $p\left((A \otimes D) \rtimes_{\mathrm{r}, 1 \otimes \alpha} \mathbb{F}_{\infty}\right) p \cong A$.

Let $B$ be a given unital separable $\mathrm{C}^{*}$-algebra. Choose a faithful state $\varphi$ on $B$. Let $\psi$ denote the state on $C([0,1])$ defined by the Riemann integral. Then by Theorem 2 of [15], the reduced free product

$$
P_{0}:=(B, \varphi) *(C[0,1], \psi)
$$


is simple. Note that by Theorem 4.8.5 of [9], the canonical inclusion $B \subset P_{0}$ admits a faithful conditional expectation (as $\psi$ is faithful). Set

$$
P:=P_{0} \otimes \mathcal{O}_{\infty}
$$

Then $P$ is unital, simple, separable, and purely infinite. The canonical inclusion $B \subset P$ still admits a faithful conditional expectation. Applying Proposition 4.4 to the trivial action of $\mathbb{F}_{\infty}$ on $P$, we obtain an (inner) action $\beta: \mathbb{F}_{\infty} \curvearrowright P$ satisfying conditions $(1)$ and (2) in the statement. Now define

$$
C:=p\left[(A \otimes D \otimes P) \rtimes_{\mathrm{r}, 1 \otimes \alpha \otimes \beta} \mathbb{F}_{\infty}\right] p .
$$

Observe that the map $x \in P \mapsto x p \in C$ defines a $\mathrm{C}^{*}$-algebra embedding. We identify $B \subset P$ with $\mathrm{C}^{*}$-subalgebras of $C$ via this embedding.

We now show that $B \subset C$ admits a faithful conditional expectation. Since $p \in D$, the canonical conditional expectation

$$
E:(A \otimes D \otimes P) \rtimes_{\mathrm{r}, 1 \otimes \alpha \otimes \beta} \mathbb{F}_{\infty} \rightarrow A \otimes D \otimes P
$$

restricts to the faithful conditional expectation $\Phi: C \rightarrow p(A \otimes D \otimes P) p$. Any faithful state $\omega$ on $p(A \otimes D) p$ induces a faithful conditional expectation $\Psi: p(A \otimes D \otimes P) p \rightarrow P$ by the formula $\Psi(p(x \otimes y) p)=\omega(p x p) y ; x \in A \otimes D, y \in P$. The composite $\Psi \circ \Phi: C \rightarrow P$ gives a faithful conditional expectation. Since $B \subset P$ has a faithful conditional expectation, consequently so does $B \subset C$.

It follows from Theorem 3.3 and [54], Lemma 5.2 that the inclusion

$$
A \cong p\left((A \otimes D) \rtimes_{\mathrm{r}, 1 \otimes \alpha} \mathbb{F}_{\infty}\right) p \subset C
$$

has no intermediate $\mathrm{C}^{*}$-algebras. By Theorem 4.8 and Lemma 4.9, the inclusion $A \subset C$ is rigid.

Remark 5.3. By a similar method to the proof of Theorem C (using [14] instead of [15]), one can confirm the following property for the free group factor $L\left(\mathbb{F}_{\infty}\right)$ : Any von Neumann algebra $M$ with separable predual embeds into a factor $N$ with a normal faithful conditional expectation which contains $L\left(\mathbb{F}_{\infty}\right)$ as a rigid maximal von Neumann subalgebra. Here we say that a von Neumann subalgebra $M \subset N$ is rigid if $\operatorname{id}_{M}$ is the only normal completely positive map $\Phi: N \rightarrow N$ satisfying $\left.\varphi\right|_{M}=\operatorname{id}_{M}$

\section{Appendix A. Tensor SPlitTing TheOREM FOR NON-UNitAl Simple $\mathrm{C}^{*}$-Algebras}

Here we record a few necessary and useful technical lemmas on non-unital $\mathrm{C}^{*}$-algebras. Although these results would be known for some experts, we do not know an appropriate reference. As a result of these lemmas, we obtain the tensor splitting theorem (cf. [18], [59], 61]) for non-unital simple $\mathrm{C}^{*}$-algebras.

An element of a $C^{*}$-algebra $A$ is said to be full if it generates $A$ as a closed ideal of $A$.

Lemma A.1. Let $A$ be a $C^{*}$-algebra. Let a be a full positive element of $A$. Then for any finite subset $F$ of $A$ and any $\epsilon>0$, there is a sequence $x_{1}, \ldots, x_{n} \in A$ satisfying

$$
\left\|\sum_{i=1}^{n} x_{i} a x_{i}^{*}\right\| \leq 1 \quad \text { and } \quad\left\|\sum_{i=1}^{n} x_{i} a x_{i}^{*} b-b\right\|<\epsilon \quad \text { for } b \in F .
$$


Proof. Observe that for any sequence $x_{1}, \ldots, x_{n} \in A$ and any $b \in F$, the $\mathrm{C}^{*}$-norm condition implies

$$
\left\|\sum_{i=1}^{n} x_{i} a x_{i}^{*} b-b\right\| \leq\left\|\sum_{i=1}^{n} x_{i} a x_{i}^{*} c-c\right\|,
$$

where $c:=\left(\sum_{d \in F} d d^{*}\right)^{1 / 2}$. Therefore we only need to show the statement when $F$ is a singleton in $A_{+}$. By the fullness of $a$, we may further assume that the element $b$ in $F$ is of the form $\sum_{i=1}^{n} y_{i} a z_{i} ; y_{1}, \ldots, y_{n}, z_{1}, \ldots, z_{n} \in A$. In this case, we have

$$
b^{2}=b^{*} b=\sum_{i, j=1}^{n} z_{i}^{*} a y_{i}^{*} y_{j} a z_{j} \leq C \sum_{i=1}^{n} z_{i}^{*} a z_{i},
$$

where $C:=\|a\|\left\|\left(y_{i}^{*} y_{j}\right)_{1 \leq i, j \leq n}\right\|_{\mathbb{M}_{n}(A)}$. Put $w:=\left(\sum_{i=1}^{n} z_{i}^{*} a z_{i}\right)^{1 / 2}$. Choose a sequence $\left(f_{k}\right)_{k=1}^{\infty}$ in $C_{0}(] 0, \infty[)_{+}$satisfying $t f_{k}(t) \leq 1$ for all $k \in \mathbb{N}$ and all $t \in\left[0, \infty\right.$, and $\lim _{k \rightarrow \infty} t f_{k}(t)=1$ uniformly on compact subsets of $] 0, \infty[$. Then, for each $k \in \mathbb{N}$, we have

$$
\begin{gathered}
\sum_{i=1}^{n} f_{k}(w) z_{i}^{*} a z_{i} f_{k}(w)=f_{k}(w)^{2} w^{2} \leq 1, \\
\left\|\sum_{i=1}^{n} f_{k}(w) z_{i}^{*} a z_{i} f_{k}(w) b-b\right\| \leq \sqrt{C}\left\|\left(w^{2} f_{k}(w)^{2}-1\right) w\right\| .
\end{gathered}
$$

The last term tends to zero as $k \rightarrow \infty$. Therefore, for a sufficiently large $N \in \mathbb{N}$, the sequence $\left(f_{N}(w) z_{i}^{*}\right)_{i=1}^{n}$ satisfies the required conditions.

For simple $\mathrm{C}^{*}$-algebras, one can strengthen Lemma A.1 as follows.

Lemma A.2. Let $A$ be a simple $C^{*}$-algebra. Let $a \in A_{+} \backslash\{0\}$. Then for any $b \in A_{+}$and any $\epsilon>0$, there is a sequence $x_{1}, \ldots, x_{n} \in A$ satisfying

$$
\left\|\sum_{i=1}^{n} x_{i} x_{i}^{*}\right\| \leq\|a\|^{-1}\|b\|, \quad \sum_{i=1}^{n} x_{i} a x_{i}^{*} \approx_{\epsilon} b .
$$

Proof. We may assume $\|a\|=\|b\|=1$. Take $f \in C([0,1])_{+}$satisfying $f(1) \neq 0$ and $\operatorname{supp}(f) \subset[1-\epsilon / 2,1]$. Then

$$
f(a) \neq 0, \quad\left(1-\frac{\epsilon}{2}\right) f(a)^{2} \leq f(a) a f(a) \leq f(a)^{2} .
$$

Applying Lemma A.1 to $f(a)^{2}$ and $F=\left\{b^{1 / 2}\right\}$, we obtain a sequence $y_{1}, \ldots, y_{n} \in A$ satisfying

$$
\left\|\sum_{i=1}^{n} y_{i} f(a)^{2} y_{i}^{*}\right\| \leq 1, \quad\left\|\sum_{i=1}^{n} y_{i} f(a)^{2} y_{i}^{*} b^{\frac{1}{2}}-b^{\frac{1}{2}}\right\|<\frac{\epsilon}{2} .
$$

Set $x_{i}:=b^{1 / 2} y_{i} f(a)$ for $i=1, \ldots, n$. Then

$$
\sum_{i=1}^{n} x_{i} x_{i}^{*}=\sum_{i=1}^{n} b^{\frac{1}{2}} y_{i} f(a)^{2} y_{i}^{*} b^{\frac{1}{2}} \leq 1 \text {. }
$$


Straightforward estimations show that

$$
\sum_{i=1}^{n} x_{i} a x_{i}^{*} \approx_{\epsilon / 2} \sum_{i=1}^{n} b^{\frac{1}{2}} y_{i} f(a)^{2} y_{i}^{*} b^{\frac{1}{2}} \approx_{\epsilon / 2} b .
$$

Therefore $x_{1}, \ldots, x_{n}$ form the desired sequence.

As an application of Lemma A.2, one can remove the unital condition from the tensor splitting theorem [59], [61] (cf. [18]). For a $\mathrm{C}^{*}$-subalgebra $C$ of $A \otimes B$, we define the subset $\mathcal{S}_{A}(C)$ of $B$ to be

$$
\mathcal{S}_{A}(C):=\left\{\left(\varphi \otimes \operatorname{id}_{B}\right)(c): \varphi \in A^{*}, c \in C\right\} .
$$

Theorem A.3. Let $A$ be a simple $C^{*}$-algebra and $B$ be a $C^{*}$-algebra. Let $C$ be a $C^{*}$ subalgebra of $A \otimes B$ closed under multiplications by $A$. Then $\mathcal{S}_{A}(C)$ forms a $C^{*}$-subalgebra of $C$ and satisfies $A \otimes \mathcal{S}_{A}(C) \subset C$. Thus, when $A$ satisfies the strong operator approximation property [25] or when the inclusion $\mathcal{S}_{A}(C) \subset B$ admits a completely bounded projection, we have $C=A \otimes \mathcal{S}_{A}(C)$.

Proof. To show the first statement, it suffices to show the following claim. For any pure state $\varphi$ on $A$ and any $a \in A_{+}, c \in C$, with $b:=\left(\varphi \otimes \operatorname{id}_{B}\right)(c)$, we have $a \otimes b \in C$. Indeed the claim implies that, since the set of pure states on $A$ spans a weak-* dense subspace of $A^{*}$ and $A_{+}$spans $A$, for any $a \in A$ and any $\psi \in A^{*}$ with $\psi(a) \neq 0$, the subspace $X:=\left(\psi \otimes \operatorname{id}_{B}\right)(C)$ of $B$ satisfies $a \otimes X=(a \otimes B) \cap C$. This implies $X=\mathcal{S}_{A}(C)$, and proves the first statement. To show the claim, for any $\epsilon>0$, by the AkemannAnderson-Pedersen excision theorem [1] (Theorem 1.4.10 in [9]), one can take $e \in A_{+}$ with $\|e\|=1$, ece $\approx_{\epsilon} e^{2} \otimes b$. By Lemma A.2, there is a sequence $x_{1}, \ldots, x_{n} \in A$ satisfying $\sum_{i=1}^{n} x_{i} e c e x_{i}^{*} \approx_{\epsilon} a \otimes b$. The left term is contained in $C$ by assumption. Thus $a \otimes b \in C$.

For the last statement, when $A$ satisfies the strong operator approximation property, the claim follows from Theorem 12.4.4 in [9]. When we have a completely bounded projection $P: B \rightarrow \mathcal{S}_{A}(C)$, it is not hard to see that for any $\varphi \in A^{*},\left(\varphi \otimes \operatorname{id}_{B}\right)\left(\left(\operatorname{id}_{A} \otimes P\right)(c)-c\right)=0$ for all $c \in C$. This proves $\left.\left(\operatorname{id}_{A} \otimes P\right)\right|_{C}=\operatorname{id}_{C}$ and thus $C \subset A \otimes \mathcal{S}_{A}(C)$.

Acknowledgements. Parts of the present work are greatly improved during the author's visiting in Research Center for Operator Algebras (Shanghai) for the conference "Special Week on Operator Algebras 2019". He is grateful to the organizers of the conference for kind invitation. This work was supported by JSPS KAKENHI Early-Career Scientists (No. 19K14550) and tenure track funds of Nagoya University. Finally, he would like to thank the second reviewer for helpful comments which improve some explanations of the article.

\section{REFERENCES}

[1] C. A. Akemann, J. Anderson, G. K. Pedersen, Excising states of $C^{*}$-algebras. Canad. J. Math., 38 (1986), 1239-1260.

[2] C. Anantharaman-Delaroche, Action moyennable d'un groupe localement compact sur une algèbre de von Neumann. Math. Scand. 45 (1979), 289-304.

[3] C. Anantharaman-Delaroche, Systèmes dynamiques non commutatifs et moyennabilité, Math. Ann. 279 (1987), 297-315.

[4] C. Anantharaman-Delaroche, Amenability and exactness for dynamical systems and their $C^{*}$ algebras. Trans. Amer. Math. Soc. 354 (2002), no. 10, 4153-4178. 
[5] P. Baum, A. Connes, N. Higson, Classifying space for proper actions and $K$-theory of group $C^{*}$ algebras. Contemp. Math. 167 (1994), 241-291.

[6] B. Blackadar, K-Theory for operator algebras. Second edition, Mathematical Sciences Research Institute Publications 5 (1998), Berkeley, CA.

[7] E. Breuillard, M. Kalantar, M. Kennedy, N. Ozawa, $C^{*}$-simplicity and the unique trace property for discrete groups. Publ. Math. I.H.É.S. 126 (2017), 35-71.

[8] L. G. Brown, G. K. Pedersen, C*-algebras of real rank zero. J. Funct. Anal. 99 (1991), 131-149.

[9] N. P. Brown, N. Ozawa, $C^{*}$-algebras and finite-dimensional approximations. Graduate Studies in Mathematics 88. American Mathematical Society, Providence, RI, 2008.

[10] I. Chifan, S. Das, Rigidity results for von Neumann algebras arising from mixing extensions of profinite actions of groups on probability spaces. Math. Ann. 378 (2020), 907-950.

[11] J. Cuntz, K-theory for certain C*-algebras. Ann. of Math. 113 (1981), 181-197.

[12] M. Dadarlat, Nonnuclear subalgebras of AF-algebras. Amer. J. Math. 122 (2000), no. 2, 581-597.

[13] M. Dadarlat, U. Pennig, A Dixmier-Douady theory for strongly self-absorbing $C^{*}$-algebras. J. reine angew. Math. 718 (2016), 153-181.

[14] K. Dykema, Factoriality and Connes' invariant $T(M)$ for free products of von Neumann algebras. J. reine angew. Math., 450 (1994), 159-180.

[15] K. Dykema, Simplicity and the stable rank of some free product $C^{*}$-algebras. Trans. Amer. Math. Soc. 351 (1999), 1-40.

[16] G. A. Elliott, G. Gong, H. Lin, Z. Niu, On the classification of simple amenable $C^{*}$-algebras with finite decomposition rank II. Preprint, arXiv:1507.03437.

[17] L. Ge, On "Problems on von Neumann algebras by R. Kadison, 1967". Acta Math. Sin. 19 (2003), no. 3, 619-624.

[18] L. Ge, R. Kadison, On tensor products of von Neumann algebras. Invent. Math. 123 (1996), 453-466.

[19] U. Haagerup, J. Kraus, Approximation properties for group $C^{*}$-algebras and group von Neumann algebras. Trans. Amer. Math. Soc. 344 (1994), 667-699.

[20] M. Hamana. Injective envelopes of operator systems. Publ. Res. Inst. Math. Sci., 15(3) (1979), 773 785.

[21] M. Hamana, Injective envelopes of $C^{*}$-algebras. J. Math. Soc. Japan 31 (1979), 181-197.

[22] M. Hamana, Injective envelopes of $C^{*}$-dynamical systems. Tohoku Math. J. (2) 37 (1985), 463-487.

[23] P. de la Harpe, G. Skandalis, Powers' property and simple $C^{*}$-algebras. Math. Ann. 273 (1986), $241-250$.

[24] N. Higson, Bivariant K-theory and the Novikov conjecture. Geom. Funct. Anal. 10 (2000), no. 3, $563-581$.

[25] N. Higson, G. Kasparov. E-theory and KK-theory for groups which act properly and isometrically on Hilbert space. Invent. Math., 144(1) (2001), 23-74.

[26] M. Izumi, R. Longo, S. Popa, A Galois correspondence for compact groups of automorphisms of von Neumann algebras with a generalization to Kac algebras. J. Funct. Anal. 155 (1998), no. 1, 25-63.

[27] M. Izumi, H. Matui, Poly-ZZZ group actions on Kirchberg algebras I. To appear in Int. Math. Res. Not., arXiv: 1810.05850 .

[28] M. Izumi, H. Matui, Poly-ZZ group actions on Kirchberg algebras II. Invent. Math. 224 (2021), 699766.

[29] M. Kalantar, M. Kennedy, Boundaries of reduced $C^{*}$-algebras of discrete groups. J. reine angew. Math. 727 (2017), 247-267.

[30] E. Kirchberg, The classification of purely infinite $C^{*}$-algebras using Kasparov's theory. Preprint.

[31] E. Kirchberg, N. C. Phillips, Embedding of exact $C^{*}$-algebras in the Cuntz algebra $\mathcal{O}_{2}$. J. reine angew. Math. 525 (2000), 17-53.

[32] A. Kishimoto, Outer automorphisms and reduced crossed products of simple $C^{*}$-algebras. Comm. Math. Phys. 81 (1981), no. 3, 429-435.

[33] A. Kishimoto, N. Ozawa, S. Sakai, Homogeneity of the pure state space of a separable $C^{*}$-algebra. Canad. Math. Bull. 46 (2003), 365-372. 
[34] E. C. Lance, Hilbert $C^{*}$-modules: a toolkit for operator algebraists. LMS Lecture Note Series 210, Cambridge University Press, Cambridge, 1995.

[35] R. Longo, Simple injective subfactors. Adv. Math. 63 (1987), 152-171.

[36] H. Matui, Y. Sato, Decomposition rank of UHF-absorbing $C^{*}$-algebras. Duke Math. J. 163 (2014), no. 14, 2687-2708.

[37] S. Neshveyev, E. Størmer, Ergodic theory and maximal abelian subalgebras of the hyperfinite factor. J. Funct. Anal., 195 (2002), no. 2, 239-261.

[38] N. Ozawa, Boundaries of reduced free group $C^{*}$-algebras. Bull. London Math. Soc. 39 (2007), 35-38.

[39] N. Ozawa, Examples of groups which are not weakly amenable. Kyoto J. Math., 52 (2012), 333-344.

[40] G. Pedersen, $C^{*}$-algebras and their automorphism groups. 2nd Edition.

[41] N. C. Phillips, A classification theorem for nuclear purely infinite simple $C^{*}$-algebras. Doc. Math. 5 (2000), 49-114.

[42] M. V. Pimsner, KK-groups of crossed products by groups acting on trees. Invent. Math. 86 (1986), no. 3, 603-634.

[43] M. V. Pimsner, A class of $C^{*}$-algebras generalizing both Cuntz-Krieger algebras and crossed products by $\mathbb{Z}$. Free probability theory, 189-212, Fields Inst. Commun., 12, Amer. Math. Soc., Providence, RI, 1997.

[44] M. Pimsner, D. Voiculescu, Exact sequences for K-groups and Ext-groups of certain cross-products of $C^{*}$-algebras. J. Operator Theory, 4 (1980), 93-118.

[45] M. Pimsner, D. Voiculescu, K-groups of reduced crossed products by free groups. J. Operator Theory 8 (1982), 131-156.

[46] S. Popa, On a problem of R.V. Kadison on maximal abelian *-subalgebras in factors. Invent. Math. 65 (1981), 269-281.

[47] S. Popa, Maximal injective subalgebras in factors associated with free groups. Adv. Math. 50 (1983), 27-48.

[48] S. Popa, Deformation and rigidity for group actions and von Neumann algebras. Proceedings of ICM 2006 Vol. I, 445-477.

[49] R. T. Powers, Simplicity of the $C^{*}$-algebra associated with the free group on two generators. Duke Math. J. 42 (1975), 151-156.

[50] M. Rørdam, Classification of nuclear, simple $C^{*}$-algebras. vol. 126 of Encyclopaedia Math. Sci., Springer, Berlin, 2002, 1-145.

[51] Y. Suzuki, Group $C^{*}$-algebras as decreasing intersection of nuclear $C^{*}$-algebras. Amer. J. Math. 139 (2017), no. 3, 681-705.

[52] Y. Suzuki, Minimal ambient nuclear $C^{*}$-algebras. Adv. Math. 304 (2017), 421-433.

[53] Y. Suzuki, Simple equivariant $C^{*}$-algebras whose full and reduced crossed products coincide. J. Noncommut. Geom. 13 (2019), 1577-1585.

[54] Y. Suzuki, Complete descriptions of intermediate operator algebras by intermediate extensions of dynamical systems. Commun. Math. Phys. 375 (2020), 1273-1297.

[55] Y. Suzuki, Rigid sides of approximately finite dimensional simple operator algebras in non-separable category. Int. Math. Res. Not. 2021 (2021), 2166-2190.

[56] Y. Suzuki, On pathological properties of fixed point algebras in Kirchberg algebras. Proc. Roy. Soc. Edinburgh Sect. A 150, Issue 6 (2020), 3087-3096.

[57] A. Tikuisis, S. White, W. Winter, Quasidiagonality of nuclear $C^{*}$-algebras. Ann. of Math. (2) 185 (2017), 229-284.

[58] W. Winter, Structure of nuclear $C^{*}$-algebras: From quasidiagonality to classification, and back again. Proc. Int. Congr. Math. (2017), 1797-1820.

[59] J. Zacharias, Splitting for subalgebras of tensor products. Proc. Amer. Math. Soc. 129 (2001), 407413.

[60] S. Zhang, A property of purely infinite simple $C^{*}$-algebras. Proc. Amer. Math. Soc. 109 (1990), 717-720.

[61] L. Zsido, A criterion for splitting $C^{*}$-algebras in tensor products. Proc. Amer. Math. Soc. 128 (2000), 2001-2006. 
Department of Mathematics, Faculty of Science, Hokkaido University, Kita 10, Nishi 8, Kita-Ku, Sapporo, HoKkaido, 060-0810, Japan

Email address: yuhei@math.sci.hokudai.ac.jp 